\title{
La maternidad: percepción universitaria sobre sus efectos en el ámbito laboral, académico y social
}

Maternity: Perception of university students about its effects in the workplace academic and social

\author{
Claudia Alejandra Hernández-Herrera ${ }^{1}$ (D https://orcid.org/0000-0002-4060-2941 \\ ${ }^{1}$ Instituto Politécnico Nacional. Correspondencia, $₫$ cahernandezh@ipn.mx \\ (C) Universidad De La Salle Bajío (México)
}

Recibido en: 26 - 06 - 2020 / Aceptado en: $18-09-2020$

\section{Resumen}

Introducción: La maternidad—uno de los roles más exigentes y no remunerados-puede ocasionar, cuando se presenta en la adolescencia, que las mujeres suspendan o abandonen sus estudios, lo que limita sus oportunidades de trabajo y desarrollo profesional, aunado al posible rechazo y discriminación de la sociedad. El objetivo de este trabajo fue analizar las percepciones que tienen los jóvenes universitarios mexicanos con respecto a la maternidad en el ámbito laboral, escolar y social.

Método: Se realizó un estudio de tipo cuantitativo. Los datos se recabaron por medio de un cuestionario con escala tipo Likert, el cual se aplicó a 2360 jóvenes de dos instituciones de educación superior de la Ciudad de México, durante los meses de septiembre a noviembre de 2017. Se empleó análisis factorial y modelos lineales generalizados.

Resultados: Se encontró que los hombres, hijos de madres que se dedicaban al hogar, fueron los que percibieron más fuerte, que la maternidad es un obstáculo para el empleo femenino. En las mujeres quienes tenían una actividad laboral, tuvieron una menor percepción de que la maternidad fuera un obstáculo para el sector laboral.

Discusión o Conclusión: Se concluye que los varones siguen manteniendo las creencias de que la maternidad es un obstáculo que corta la vida laboral de las mujeres, debido a que ellos continúan atribuyéndoles a ellas la responsabilidad de la crianza de los hijos, así como la prevención del embarazo.

Palabras clave: maternidad; estudiantes; educación superior; discriminación; percepción; jóvenes; vida laboral; mujeres; embarazos; crianza; roles de género; empleo femenino 


\begin{abstract}
Introduction: Maternity (one of the roles most demanding and least valued by society) can cause that women interrupt or drop out their studies-which limits their job and professional development opportunities, coupled with the possible rejection and discrimination from society. The aim of this work was to analyze the perceptions that Mexican young undergraduates have regarding maternity in the workplace and school. A quantitative study was conducted.

Method: Data were collected through a Likert-type survey questionnaire, which was applied to 2 360 young people from two institutions of higher education in Mexico City, during the months of September to November 2017. Factor analysis and generalized linear models were used.

Results: It was found that the sons of mothers dedicated to their housework were the ones who perceived strongly that maternity is an obstacle for female employment. In the case of women, the ones who had a work activity showed a lower perception that maternity was an obstacle for the working sector.

Discussion or Conclusion: It is concluded that men continue to maintain beliefs that motherhood is an obstacle, and that it cuts the working life of women - that is because men continue to attribute to women the responsibility of raising children as well as of preventing pregnancies.
\end{abstract}

Keywords: motherhood; students; higher education; discrimination; perception; youths; laboral life; women; pregnancies; breeding; gender roles; female employment

\title{
Introducción
}

De acuerdo con la feminista Simone De Beauvoir (1981) psicológicamente la maternidad puede ser provechosa pero también amarga, las consecuencias del parto pueden acarrear dificultades y en ocasiones serias enfermedades, aunado a los cuidados que integra la posible lactancia la cual es agotadora y demandante. Además, la maternidad orilla a la mujer a una vida sedentaria que le pone obstáculos para su realización personal e intelectual, por ende, dificulta su acceso a los espacios de trabajo que les permitan poseer derechos y libertad económica. Por lo anterior, estudiar las percepciones de los jóvenes con respecto a las creencias sobre la maternidad es importante, ya que, de acuerdo con Petit y Graglia (2005) las percepciones humanas son construcciones que se encuentran activas todo el tiempo y dependen de los valores, las creencias y las representaciones sociales, con lo que se interpreta la realidad y el mundo. Además, Ibáñez (2011) señala que la 
percepción es todo aquel proceso mediante el cual los individuos obtiene información del entorno que los rodea, haciendo uso de sus sentidos. El percibir a las personas implica tareas de clasificación, atribución de características y de significados los cuales son sociales derivados de las interacciones que tenemos con los demás, producto de igual forma de la historia de los grupos sociales a los que pertenecen las personas. Los elementos anteriormente expuestos, permiten conocer los factores que influyen en las formas en cómo los estudiantes interpretan el fenómeno de la maternidad.

También, la maternidad ha sido usada por el patriarcado como el destino de las mujeres y de esa forma relegarlas al ámbito doméstico y privado que las convierte en seres invisibles. Entonces la maternidad es un fenómeno complejo de explicar, sin embargo los temas de elección y control de la sexualidad de las mujeres son factores que permiten tomar decisiones con respecto a su cuerpo. De acuerdo con Donath (2017) la maternidad puede ser tan difícil que origina arrepentimiento en muchas mujeres. Por lo anterior, García (2015) enfatiza que la maternidad continúa estando pendiente de soluciones, ya que las mujeres necesitan deconstruir la maternidad y reconstruirla sobre un mundo completamente distinto. Sin embargo, es evidente que algunas mujeres continúan luchando para estar presentes en los espacios educativos y laborales, pero el camino es complejo, el rol de la maternidad exige cuidados y tiempo.

El embarazo cuando se presenta en edades tempranas, puede provocar complicaciones en la salud, aunado a que trae consigo serias repercusiones, como una menor escolaridad, escasa asistencia a la escuela, la poca presencia en los mercados laborales, y por ende menores ingresos en las familias (Arceo-Gómez y Campos-Vazquez, 2014). Se requiere aclarar que el embarazo se convierte en un problema cuando éste no es planeado o no deseado, lo que desencadena una serie de situaciones que orillan a las mujeres a tomar decisiones bajo mucha presión social y familiar, aunada a que los espacios educativos y laborales condicionan su permanencia, coartando sus oportunidades de progreso y desarrollo.

De acuerdo con Frías (2011) las mujeres sufren discriminación, porque las empresas acostumbran exigir pruebas de no embarazo en el proceso de reclutamiento, y cuando se incumple el requisito son por completo descartadas. Ahora bien, la Ley Federal del Trabajo solo protege a las mujeres contratadas, no durante el proceso de contratación. La discriminación por razones de embarazo sigue estando presente a pesar de que México ratificó tratados y convenciones internacionales que exhortan a los países a garantizar la protección de las mujeres hacia este 
fenómeno; 1) la Convención 111 de la Organización Internacional del Trabajo, 2) la Convención sobre la Eliminación de todas las formas de Discriminación contra la Mujer (CEDAW), 3) la Convención Americana de los derechos Humanos y 4) la Convención Internacional de Derechos Civiles y Políticos.

Ahora bien, la Convención sobre la eliminación de todas las formas de discriminación contra la mujer (1979) señala que los países requieren trabajar para modificar los patrones socioculturales de comportamiento de hombres y mujeres, de tal forma que se logren eliminar los prejuicios de inferioridad y superioridad. Otra de las situaciones a las que se enfrentan las mujeres madres de familia, es encontrar trabajos permanentes y de tiempo completo cuando sus hijos son pequeños, lo que provoca que sus ingresos sean bajos (Ezawa, 2007). Es evidente que el mercado de trabajo se divide en empleos parentales y no parentales (Yerkes, 2010). Existen hallazgos que muestran que, cuando se retarda la maternidad, en las mujeres incrementa la posibilidad para emplearse de tiempo completo y alcanzar niveles altos de educación (Stafford et al., 2018). En contraparte, se encontró que las mujeres con hijos, que usaron el autoempleo, obtuvieron mayores ingresos y tuvieron más empleados en sus empresas (Lappegård y Rønsen, 2005; Joona, 2018).

Otra de las variables que marcan el fenómeno es la brecha salarial, que muestra que las mujeres sin hijos perciben mayores ingresos que sus pares que tienen hijos. Y a mayor edad de las mujeres, la penalidad salarial por maternidad disminuye, pero influye el nivel de escolaridad. Por otro lado, el tema de los cuidados en ocasiones conlleva que las horas que destinan los hombres al trabajo doméstico, a pesar de que estas vayan en aumento, no tienen implicaciones directas en los ingresos de las mujeres, ya que ellos evitan actividades que incluyan el cuidado de los niños (Gash, 2009; Felfe, 2012; Staff y Mortimer, 2012; Gamboa y Zuluaga, 2013; Viitanen, 2014; De-Hoon et al., 2017; Li, 2017; Morgenroth y Heilman, 2017).

Ahora bien, otra situación que se presenta es que la mayoría de las mujeres que son madres jóvenes abandonan la escuela, entonces a bajo nivel de educación, sus posibilidades de ingresar a un empleo digno son escasas, y la falta de dinero produce más pobreza y violencia hacia ellas, además, el abandono de la pareja provoca que ellas se hagan responsables de la doble crianza (Allen et al., 2012; Baranowska-Rataj et al., 2014; Watts et al., 2015; Rodríguez-Vignoli, 2017). Además, las estudiantes que son madres en ocasiones obtienen resultados académicos deficientes, sufren carencias económicas y sus hijos llegan a presentar dificultades en la escuela (Breen y McLean, 2010). De acuerdo con Narita y Díaz (2016), cuando una mujer joven vive en lugares que presentan 
pobreza, no desarrolla expectativas sobre su futuro, entonces desdibujan sus posibilidades académicas y laborales, y la falta de un plan de vida las lleva a tomar decisiones radicales sobre su sexualidad y el embarazo (Berthelon y Kruger, 2017).

En México, se identificó estudios con relación al embarazo en adolescentes y el rezago educativo, en los que se encontró que, las jóvenes presentan ausencia en la escuela y pertenecen a niveles socioeconómicos bajos, por lo que se requieren medidas emergentes que favorezcan la permanencia de las mujeres en las aulas, con y sin hijos (Villalobos-Hernández et al., 2015). Por otra parte, investigaciones realizadas con mujeres universitarias, se les cuestionó sobre temas relacionados con la carrera, el matrimonio y la maternidad, y se encontró que, siete años después del egreso de la carrera, las mujeres que eran madres, fueron quienes habían alcanzado menos títulos académicos y un menor avance en sus carreras, que las mujeres que no tenían hijos (Hoffnung, 2004). Existen hallazgos de que el retraso de la maternidad en la adolescencia favorece los resultados en la carrera y en los ingresos (Amuedo-Dorantes y Kimmel, 2005; Miller, 2011; Looze, 2014; Myrskylä et al., 2017). Además, de que el incremento de las aspiraciones académicas aparece cuando la maternidad se retrasa (Devos et al., 2007).

Se han planteado acciones para que los jóvenes cuenten con información para prevenir el embarazo y se ocupen de sus metas por medio del desarrollo personal y la autoestima (Atienzo et al., 2014). Además, se han analizado reflexiones de estudiantes con relación a las prácticas de la maternidad y la paternidad durante la etapa de estudiantes, hallándose que las mujeres se enfrentan a los problemas que implica conciliar las actividades de ser cuidadoras y estudiantes; con los varones se halló que ellos afrontan el papel de ser padres y proveedores (Stadelmann, 2011; Drange y Rege, 2013; Sánchez, 2015).

Por otra parte, Weed y Nicholson (2015), encontraron que los jóvenes universitarios perciben negativamente a las estudiantes que son madres o están embarazadas, ya que se tiene la creencia de que son personas irresponsables. Lo preocupante es que al parecer la maternidad es una nueva forma de división social entre las mujeres (Thomson, 2014; Berthelon y Kruger, 2017). De acuerdo con Behboodi et al. (2017), en ocasiones, las madres jóvenes enfrentan en la escuela comentarios discriminatorios, aunado a las presiones emocionales y las cargas de trabajo doméstico. Al respecto, se tiene evidencia de que las madres estudiantes se sienten rechazadas en el ambiente universitario, por lo que su salud emocional se deteriora (Aponte y Correa, 2012). 
Por último, de acuerdo con Behboodi et al. (2017), las instituciones educativas olvidan que la maternidad es un fenómeno que está presente, y las universidades no han conseguido adaptarse, $\mathrm{y}$, por lo tanto, no llegan a considerarlo un tema transcendental que requiere ocupar un lugar en las agendas de trabajo. Por lo anterior, es necesario poner en la mesa, que los procesos de reproducción biológicos de las mujeres en ocasiones no empatan con los procesos académicos que se dan en sistemas educativos poco flexibles y con diseños curriculares que no son apropiados, lo que en ocasiones no permite que ellas desarrollen su potencial. Por lo anterior, surge la pregunta de investigación que se aborda en el artículo ¿cuáles son los factores que inciden con mayor frecuencia en la percepción de los universitarios mexicanos con relación a las creencias que rodean al fenómeno de la maternidad en el ámbito laboral, académico y social?

El estudiar las percepciones de los jóvenes universitarios mexicanos permite conocer las interpretaciones que le otorgan los estudiantes al fenómeno de la maternidad para identificar los avances, retrocesos y de esa forma visibilizar a la maternidad como un rol complejo con implicaciones en ocasiones difíciles que frena el desarrollo de las mujeres que son madres en el ámbito laboral, académico y social. El objetivo del artículo fue estudiar las percepciones de jóvenes universitarios con relación a las creencias sobre maternidad y los elementos que se vislumbran en el sector laboral, el académico y el social para identificar las posibles variaciones de las variables contextuales y determinar los avances, retrocesos con respecto al fenómeno para así descubrir las brechas que existen entre las formas de pensamiento de las mujeres y hombres estudiantes. Lo anterior, se realizó mediante los modelos lineales generalizados que permiten efectuar análisis multivariante y de esa forma inferir considerando las categorías de las variables sociodemográficas de los jóvenes.

\section{Método}

Se realizó un estudio transversal descriptivo. Para ello, se diseñó y validó un instrumento que mide las percepciones de los estudiantes universitarios con relación al fenómeno de la maternidad. La confiabilidad del cuestionario se trabajó a partir del alfa de Cronbach, cuyo resultado fue de 0.820. El instrumento se integró por 21 ítems con escala tipo Likert, en donde $1=$ totalmente en desacuerdo, 2 = en desacuerdo, $3=$ ni de acuerdo ni en desacuerdo, $4=$ de acuerdo y $5=$ totalmente de acuerdo. Para la estructura de los enunciados, se realizó una revisión de la literatura, la búsqueda de los artículos se llevó a cabo tomando en cuenta motores de búsqueda relacionados con la 
maternidad y la juventud, la maternidad y la universidad, la maternidad y el mercado laboral. El análisis de los artículos permitió la estructura de los enunciados que integran el instrumento. El cuestionario fue piloteado con un grupo de 60 estudiantes de una de las unidades académicas participantes en el estudio, lo que permitió conocer el comportamiento de las variables y las pruebas estadísticas.

Posteriormente, se realizó un análisis de factores para identificar la capacidad de agruparse por el método de componentes principales y rotación varimax. La prueba de Kaiser Meyer Olkin fue de $0.837>0.50$. Se obtuvieron 5 factores del análisis factorial exploratorio, siendo el número 1, la maternidad y el empleo femenino, que representa el mayor porcentaje de la varianza, con el $22.44 \%$; el segundo, la maternidad y el entorno académico; el tercero, la maternidad y los programas sociales; el cuarto, la universidad y los programas de apoyo orientados a la maternidad, y el quinto, creencias sobre el aborto (Tabla 1). Se realizó un análisis factorial y se aplicaron los modelos lineales generalizados con los 5 factores. Para determinar las diferencias con respecto a las variables independientes, se tomó la decisión de dividir el modelo en 2 partes, ya que técnicamente la cantidad de datos es extensa. Se trabajó con la prueba estadística de ómnibus por cada modelo lineal generalizado; esta prueba permite identificar la existencia de variación del factor y las variables predictoras del modelo, para así determinar si éstas coadyuvan a explicar la variable criterio. Para cada 1 de los 5 modelos lineales que se trabajaron, se decidió ocupar, para la primera parte, las variables de la escolaridad y la ocupación de la madre, la escolaridad y ocupación del padre y si se tenían hijos. Para la segunda parte, se trabajó con las variables que miden la existencia de vida sexual de parte de los jóvenes, si utilizaban un método anticonceptivo, el tipo de método anticonceptivo, si contaban con trabajo, percepción sobre la edad ideal para convertirse en madre, saber si la escuela proporcionaba anticonceptivos, y dinero que se gastaba al mes para adquirir anticonceptivos. Se trabajó la prueba de rachas, para dividir la base de datos por género en SPSS.

Además, se aplicó la prueba no paramétrica de U de Mann Whitney entre los grupos formados por las mujeres y los hombres, para identificar la posible diferencia o similitud de los rangos promedios obtenidos.

Los cuestionarios se aplicaron a 2360 alumnos, de 2 escuelas de educación superior públicas, pertenecientes al Instituto Politécnico Nacional y al Tecnológico Nacional de México, ambas ubicadas en la Ciudad de México. 
Tabla 1. Análisis factorial, reducción de dimensiones por componentes principales.

Table 1. Factorial analysis, reduction of dimensions by main components.

\section{Dimensión 1. La maternidad y empleo. \\ Coeficiente de correlación \\ Estudios}

12. Creo que el mercado laboral favorece a las mujeres que no tienen hijos sobre las que si los tienen.

13. El actual mercado de trabajo por su nivel de competencia no contempla ofrecer oportunidades de empleo a las madres jóvenes.

11. Creo que las madres jóvenes no tienen empleo porque es evidente su falta de capacidad y tiempo para dedicarse al trabajo.

4. Las mujeres que no tienen hijos se destacan en su profesión y se refleja en su remuneración.

5. Es una realidad, que la mayoría de las mujeres embarazadas o madres jóvenes dependen económicamente de sus familias.

20. Pienso que cada vez es más frecuente que las empresas soliciten análisis de sangre para detectar embarazo.

21. Es más frecuente que pierdan su empleo las mujeres jóvenes que se embarazan.

\section{Dimensión 2. La maternidad y el entorno académico}

10. En la escuela, es evidente el abandono de los estudios universitarios de parte de las madres solteras.

2. Las mujeres universitarias que son madres o están embarazadas, a pesar de contar con las habilidades e inteligencia, es difícil que se distingan en clase, ya que es evidente su falta de tiempo.

1. Las mujeres universitarias que se embarazan cortan su futuro profesional.

9. Creo que existen algunos profesores que discriminan a las estudiantes por estar embarazadas.

16. Considero que en su mayoría las mujeres jóvenes embarazadas sufren abandono por parte de su pareja.

\section{Dimensión 3. La maternidad y los programas sociales}

18. Creo que es necesario que el gobierno se haga cargo de la asistencia médica de las madres jóvenes y por ende de sus hijos.

19. Me parece que es necesario que las empresas mantengan políticas de conciliación, que les permitan a las mujeres embarazadas cuidar de ellas y de sus hijos.

17. Las mujeres jóvenes que están embarazadas necesitan el apoyo del gobierno federal, el cual, les permita emprender un negocio.

6. Conozco los programas sociales del gobierno federal a las que pueden acceder las madres solteras.

Dimensión 4. La universidad y los programas de apoyo orientados a la maternidad

7. En la escuela de forma continua se realizan campañas para la prevención del embarazo.

8. En la escuela se tienen programas para apoyar a las madres que no tienen en dónde dejar a sus hijos.

3. Las mujeres que están embarazadas en todo momento están protegidas por la escuela, ésta las orienta y las apoya.

\section{Dimensión 5. Creencias sobre el aborto}

15. Considero que las mujeres que abortan deben ser castigadas por la ley por considerarse un delito.

14. Creo que las mujeres jóvenes no tienen derecho de abortar, porque fue su responsabilidad el quedar embarazadas y por lo tanto deben asumirla.
0.663

0.662

0.614

0.585

0.527

(Noonan et al., 2007; Yerkes, 2010; Casas y Herrera, 2012; Pacelli, et al., 2013; Looze, 2014; Apps et al., 2016; Lavee, y Benjamin, 2016; Grunow y Aisenbrey, 2016; Ciciolla et al., 2017; De-Hoon, et al., 2017).

Coeficiente de correlación

0.699

Looze, 2014; ArceoGómez y CamposVázquez, 2014, Weed y 0.675 Nicholson, 2015;

$0.528 \quad$ Behboodi, et al., 2017; Berthelon y Kruger, 2017; $0.510 \quad$ Watson y Vogel, 2017).
0.800

0.734

(Giddings et al., 2004;

\section{Coeficiente de} correlación
0.766

0.733

(Ezawa, 2007; Drange y Rege, 2013; Li et al., 2017).

0.598

\section{Coeficiente de} correlación

0.870

0.869

(Watts et al., 2015)

Fuente: Elaboración propia a partir de los resultados obtenidos del software SPSS.

Source: Own construction from the results obtained from the SPSS software.

No 26, Vol. 13 (2), 2021. ISSN 2007 - 0705, pp.: 1 - 29

$$
-8 \text { - }
$$




\section{$\underline{\text { Resultados }}$}

De los 2360 cuestionarios, el $51 \%$ fueron mujeres y el $49 \%$ hombres. De acuerdo a la respuesta de los jóvenes, el nivel de escolaridad de la mamá se distribuye de la siguiente manera: 4 \% no tenía estudios, $12 \%$ contaba con primaria concluida, $30 \%$ con secundaria, $35 \%$ con preparatoria, $18 \%$ con licenciatura y solo el $1 \%$ tenía un posgrado. En cuanto a la ocupación de la mamá, el 46 $\%$ de ellas se dedicaba al hogar, el $27 \%$ trabajaba por su cuenta, el $15 \%$ trabajaba para una empresa y el $12 \%$ para el gobierno. Sobre la escolaridad del padre, el $5 \%$ de ellos no tenía estudios, el $9 \%$ contaba con primaria concluida, el $23 \%$ con secundaria, el $35 \%$ con preparatoria, el $24 \%$ con licenciatura y un $4 \%$ tenía posgrado. Con respecto a su ocupación, el $41 \%$ trabajaba por su cuenta, el $35 \%$ para una empresa, el $15 \%$ laboraba para el gobierno y el $9 \%$ se dedicaba al hogar.

El $93 \%$ de los jóvenes estudiantes encuestados dijo no tener hijos, y el 60 \% enfatizó mantener una vida sexual activa; de ellos, el $63 \%$ de los varones la tenía, y en el caso de las mujeres, un $57 \%$. Aunado a lo anterior, de los jóvenes que dijeron mantener una vida sexual activa el $91.89 \%$ de los hombres manifestó que ellos o sus parejas usaban algún método anticonceptivo, mientras que solo el $81.75 \%$ de las mujeres señaló que ellas o sus parejas lo usaban también. Con relación al tipo de método anticonceptivo empleado se realizó el cruce de la variable que mide si tienen vida sexual activa, y se encontró que el $87 \%$ de los hombres utilizaba el preservativo, y el $75 \%$ de las mujeres dijo que su pareja lo empleaba. Por otro lado, el $9.46 \%$ de las mujeres tomaba pastillas, y el $3.09 \%$ de los varones dijo que sus parejas las tomaban también; en cuanto a las inyecciones, solo el $3.39 \%$ de ellas revelaron que las utilizaban, mientras que el $3.25 \%$ de los hombres indicaron que sus parejas también las usaban; con relación al método del ritmo, se encontró que el $2.85 \%$ de las mujeres señalaron que lo practicaban, y el $2.01 \%$ de los varones de igual forma afirmaron emplearlo. Por otro lado, el $9.30 \%$ de las mujeres manifestaron utilizar otros tipos de anticonceptivos, mientras que en el caso de los varones se halló que el $4.65 \%$, de igual forma ocupan otros anticonceptivos.

En cuanto a la edad que los estudiantes consideran que es la ideal para convertirse en mamá, el $41 \%$ dijo que, entre los 28 y 31 años, el $33 \%$ señaló que de los 24 a los 27 años, el $15 \%$ de los 32 a los 35 años, el $4 \%$ entre los 20 a los 23 años, el $3 \%$ entre los 36 y los 39 años y el $4 \%$ más de 40 años. Se cuestionó a los alumnos si la escuela les proporcionaba anticonceptivos (únicamente se entregan preservativos), el $66 \%$ dijo que si, mientras el resto dijo que no. 


\section{Factor 1. La maternidad y el empleo femenino}

En este factor se integran los elementos de análisis que permiten conocer el sentir de los jóvenes con aquellas percepciones que reflejan a la maternidad como un obstáculo que limita y corta el futuro profesional de las mujeres. De acuerdo a la prueba de U de Mann Whitney, en los grupos formados por las mujeres y los hombres, se encontró diferencia $(\mathrm{P}<0.05)$ entre ellos. Los hombres obtuvieron mayor rango promedio. De igual forma se corrió la prueba con los grupos formado por los grupos de los estudiantes que dijeron tener hijos y los que no, los resultados arrojaron que no existe diferencia entre ellos $(\mathrm{P}>0.05)$ (Tabla 2). En cuanto a los modelos lineales generalizados, la prueba de contraste de Omnibus, en la primera parte, obtuvo que el grupo de los hombres se tienen variables con efectos hacia el modelo $(\mathrm{P}<0.05)$, mientras que en el grupo de las mujeres no se encontró. En la prueba de los efectos del modelo, en el factor la maternidad y empleo femenino, en el caso de los varones, las variables que aportan a la explicación del factor son; la ocupación de la madre y la ocupación del padre $(\mathrm{P}<0.05)$ (Tabla 3). De acuerdo con las medias marginales, se halló que los hombres que dijeron que sus madres trabajan por su cuenta (26.57) y que se dedican al hogar (25.99), fueron quienes percibieron más alto este factor. En el caso de la variable ocupación del padre, los varones que dijeron que sus padres que se dedicaban al hogar fueron los que le dieron mayor valor a las creencias que pueden poner en desventaja a las mujeres en el mercado de trabajo (29.31), mientras que aquellos varones cuyos padres trabajaban en el gobierno les otorgaron menos valor a estas creencias (25.46) (Tabla 4).

En la parte dos, del modelo lineal generalizado, referente a la variable de la existencia de vida sexual activa en los jóvenes, la prueba de contraste de Omnibus encontró que en el grupo de mujeres y de hombres se tienen variables que coadyuvan al análisis del factor $(\mathrm{P}<0.05)$.

En los efectos del modelo, en el caso de los hombres, se hallaron que las variables de utilización de métodos anticonceptivos, tipo de método anticonceptivo más usado, percepción sobre a qué edad es adecuado convertirse en madre, la escuela provee de anticonceptivos y cantidad del dinero al mes que se gasta en anticonceptivos aportan información al factor que analiza la maternidad y el empleo femenino (Tabla 3). Se detectó que aquellos que calificaron alto las creencias sobre la maternidad y el empleo fueron quienes expresaron que no usaban métodos anticonceptivos (29.70); lo mismo ocurre con el grupo de hombres que dijeron que si empleaban algún método anticonceptivo (él o sus parejas) y que preferían las inyecciones (31.25); y los que consideraron que la edad adecuada para ser madre es de más de 40 años (31.20). De igual manera, 
calificaron alto los que dijeron que la escuela no provee anticonceptivos (27.40), y en el caso de aquellos jóvenes que los usaban, manifestaron gastar unos 250 pesos mexicanos mensuales en ellos (29.11).

En el caso de las mujeres, se encontró que la variable de si cuenta con empleo es la que aporta al factor $(\mathrm{P}<0.05)$. Hallándose que de acuerdo con los resultados de las medias se obtuvo que aquellas jóvenes que no trabajaban durante el estudio fueron quienes calificaron más alto las creencias de maternidad y el mercado laboral (24.85), caso contrario sucedió con las estudiantes que han trabajado (23.96), ya que su percepción disminuyó con relación a las creencias mencionadas (Tabla 4).

Tabla 2. Prueba de U de Mann Whitney de los cinco factores estudiados.

Table 2. Mann Whitney U test of five factors studied.

\begin{tabular}{|c|c|c|c|c|}
\hline & $\begin{array}{c}\text { Mujeres } \\
\text { (N = 1 222) } \\
\text { Rangos } \\
\text { promedios }\end{array}$ & $\begin{array}{c}\text { Hombre } \\
(\mathbf{N}=1 \text { 138) } \\
\text { Rangos promedios }\end{array}$ & $\begin{array}{c}\text { Si tienen } \\
\text { hijos } \\
\text { Rangos } \\
\text { promedios }\end{array}$ & $\begin{array}{l}\text { No tienen } \\
\text { hijos } \\
\text { Rangos } \\
\text { promedios }\end{array}$ \\
\hline Maternidad y empleo & $1091.07^{\mathrm{a}}$ & $1223.82^{b}$ & $1089.13^{\mathrm{a}}$ & $1178.94^{\mathrm{a}}$ \\
\hline $\begin{array}{c}\text { Maternidad y entorno } \\
\text { universitario }\end{array}$ & $1089.77^{a}$ & $1216.29^{b}$ & $946.31^{\mathrm{a}}$ & $1184.29^{\mathrm{b}}$ \\
\hline $\begin{array}{c}\text { Maternidad y programas } \\
\text { sociales }\end{array}$ & $1134.82^{\mathrm{a}}$ & $1180.92^{\mathrm{a}}$ & $1261.94^{\mathrm{a}}$ & $1168.86^{\mathrm{a}}$ \\
\hline $\begin{array}{c}\text { Universidad y programas } \\
\text { de apoyo orientadas a la } \\
\text { maternidad }\end{array}$ & $1096.73^{\mathrm{a}}$ & $1220.78^{a}$ & $1048.55^{\mathrm{a}}$ & $1183.40^{b}$ \\
\hline $\begin{array}{l}\text { Las creencias sobre el } \\
\text { aborto }\end{array}$ & $1099.70^{\mathrm{a}}$ & $1222.82^{\mathrm{a}}$ & $1165.79^{\mathrm{a}}$ & $1177.80^{\mathrm{a}}$ \\
\hline
\end{tabular}

${ }^{\mathbf{a}, \mathbf{b}}$ Letras distintas indican diferencia significativa $(\mathrm{P}<0.01)$.

a, $\mathbf{b}$ The different letters indicate significant difference $(\mathrm{P}>0.01)$.

Fuente: Elaboración propia a partir de los resultados obtenidos del software SPSS.

Source: Own construction from the results obtained from the SPSS software. 
Tabla 3. Prueba de los efectos del modelo lineal generalizado.

Table 3. Effects of the generalized linear model test.

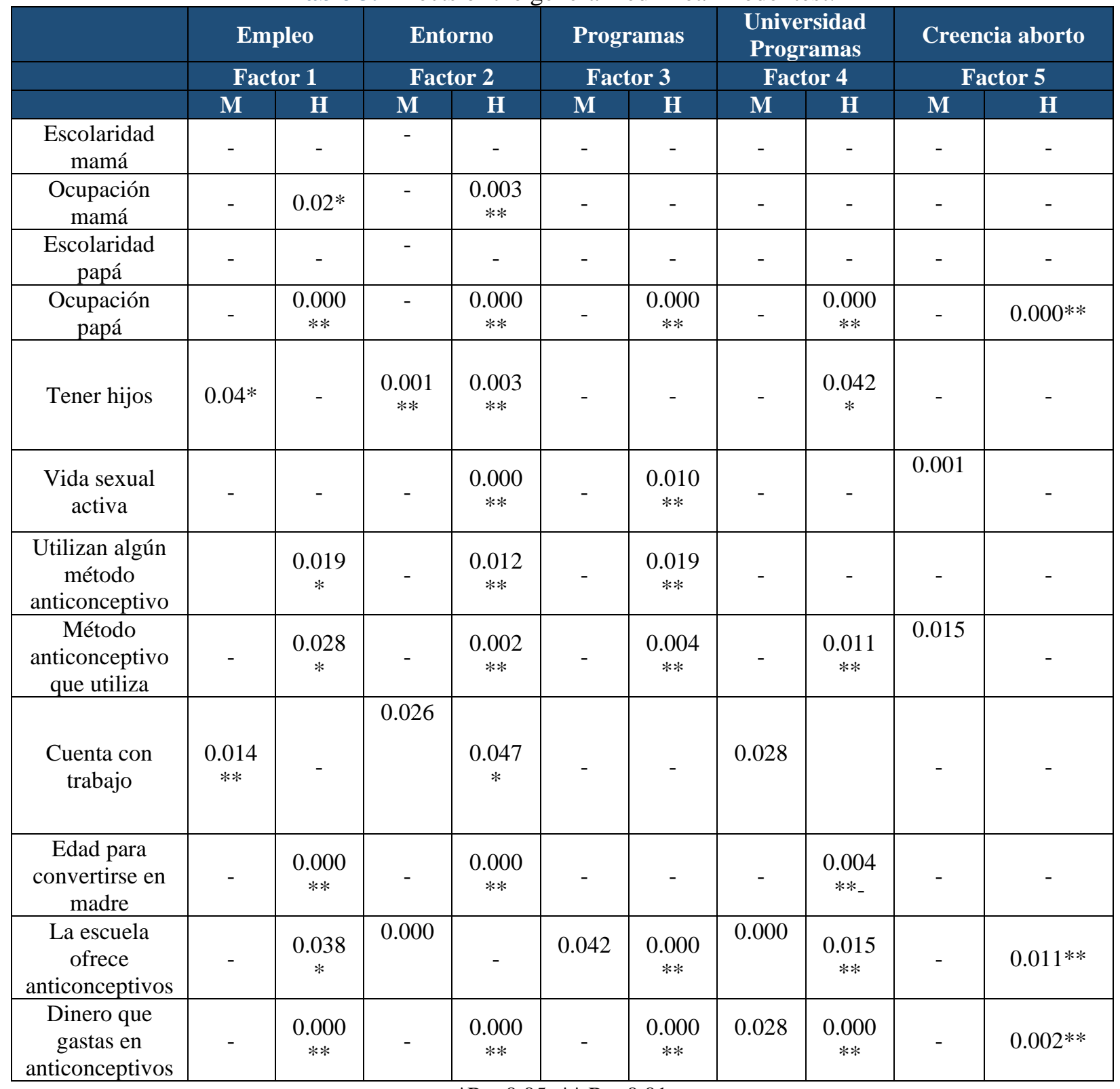

$* \mathrm{P}<0.05 ; * * \mathrm{P}<0.01$

Fuente: Elaboración propia a partir de los resultados obtenidos del software SPSS. Source: Own construction from the results obtained from the SPSS software. 
Tabla 4. Rangos promedios de las categorías de las variables predictoras de los factores.

Table 4. Average ranges for the categories of the predictor variables for the factor.

\begin{tabular}{|c|c|c|c|c|c|c|c|c|c|c|c|}
\hline \multirow[b]{3}{*}{ Variables } & \multicolumn{5}{|c|}{ Mujeres } & & \multicolumn{5}{|c|}{ Hombres } \\
\hline & \multicolumn{5}{|c|}{ Factores } & & \multicolumn{5}{|c|}{ Factores } \\
\hline & 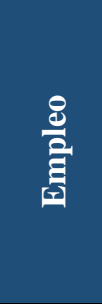 & 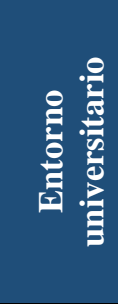 & 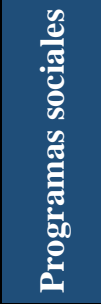 & 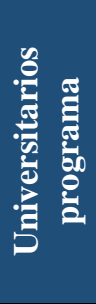 & 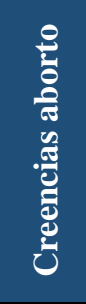 & Variables & $\frac{8}{0}$ & 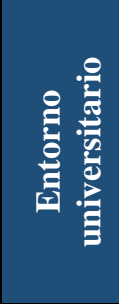 & 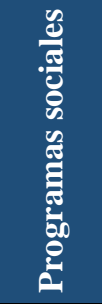 & 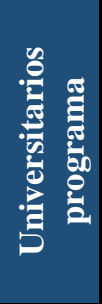 & 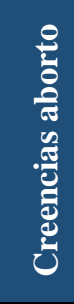 \\
\hline \multicolumn{6}{|c|}{ Escolaridad de la mamá } & \multicolumn{6}{|c|}{ Escolaridad de la mamá } \\
\hline $\begin{array}{l}\text { No tiene } \\
\text { estudios }\end{array}$ & 21.12 & 23.08 & 14.35 & 8.30 & 4.15 & $\begin{array}{l}\text { No tiene } \\
\text { estudios }\end{array}$ & 24.33 & 26.82 & 13.67 & 9.51 & 6.08 \\
\hline Primaria & 22.81 & 23.89 & 15.11 & 8.50 & 4.19 & Primaria & 24.35 & 26.51 & 14.27 & 9.54 & 6.60 \\
\hline Secundaria & 22.19 & 24.05 & 14.68 & 8.84 & 4.20 & Secundaria & 24.72 & 27.13 & 14.36 & 9.85 & 6.25 \\
\hline Preparatoria & 22.01 & 23.99 & 14.80 & 8.76 & 3.96 & Preparatoria & 24.67 & 27.09 & 14.60 & 9.79 & 6.07 \\
\hline Licenciatura & 22.28 & 23.85 & 14.79 & 8.81 & 3.79 & Licenciatura & 24.38 & 26.97 & 14.33 & 9.65 & 5.69 \\
\hline Posgrado & 21.90 & 22.62 & 16.53 & 8.82 & 3.62 & Posgrado & 23.48 & 25.07 & 13.80 & 8.71 & 5.04 \\
\hline \multicolumn{6}{|c|}{ Ocupación de la madre } & \multicolumn{6}{|c|}{ Ocupación de la madre } \\
\hline $\begin{array}{c}\text { Trabaja en } \\
\text { una empresa }\end{array}$ & 21.88 & 22.73 & 14.44 & 8.10 & 3.65 & $\begin{array}{c}\text { Trabaja en } \\
\text { una empresa }\end{array}$ & 25.47 & 28.11 & 15.24 & 9.93 & 6.71 \\
\hline $\begin{array}{c}\text { Trabaja en el } \\
\text { gobierno }\end{array}$ & 21.45 & 22.88 & 14.10 & 8.04 & 3.52 & $\begin{array}{c}\text { Trabaja en el } \\
\text { gobierno }\end{array}$ & 25.81 & 28.64 & 15.10 & 9.95 & 6.37 \\
\hline $\begin{array}{c}\text { Trabaja por } \\
\text { su cuenta }\end{array}$ & 21.30 & 22.57 & 14.34 & 8.14 & 3.60 & $\begin{array}{l}\text { Trabaja por } \\
\text { su cuenta }\end{array}$ & 26.57 & 29.56 & 15.56 & 10.32 & 6.42 \\
\hline $\begin{array}{c}\text { Se dedica al } \\
\text { hogar }\end{array}$ & 21.75 & 22.68 & 14.33 & 8.05 & 3.96 & $\begin{array}{c}\text { Se dedica al } \\
\text { hogar }\end{array}$ & 25.99 & 28.30 & 15.60 & 10.03 & 6.36 \\
\hline \multicolumn{6}{|c|}{ Escolaridad del padre } & \multicolumn{6}{|c|}{ Escolaridad del padre } \\
\hline $\begin{array}{l}\text { No tiene } \\
\text { estudios }\end{array}$ & 22.69 & 24.11 & 14.81 & 9.25 & 4.20 & $\begin{array}{l}\text { No tiene } \\
\text { estudios }\end{array}$ & 24.10 & 25.86 & 14.68 & 9.50 & 6.31 \\
\hline Primaria & 21.07 & 23.04 & 15.06 & 8.36 & 3.87 & Primaria & 25.35 & 28.07 & 14.74 & 10.04 & 6.02 \\
\hline Secundaria & 21.96 & 22.79 & 15.00 & 8.28 & 3.92 & Secundaria & 24.65 & 27.92 & 14.28 & 10.27 & 5.70 \\
\hline Preparatoria & 22.45 & 22.98 & 14.67 & 8.42 & 3.69 & Preparatoria & 24.69 & 27.80 & 14.28 & 10.11 & 5.69 \\
\hline Licenciatura & 21.84 & 22.72 & 14.83 & 8.29 & 3.70 & Licenciatura & 25.23 & 28.01 & 14.26 & 10.28 & 5.78 \\
\hline Posgrado & 22.43 & 23.82 & 14.46 & 8.52 & 3.34 & Posgrado & 26.12 & 28.76 & 14.22 & 10.13 & 6.36 \\
\hline \multicolumn{6}{|c|}{ Ocupación del padre } & \multicolumn{6}{|c|}{ Ocupación del padre } \\
\hline $\begin{array}{c}\text { Trabaja en } \\
\text { una empresa }\end{array}$ & 22.34 & 23.48 & 14.64 & 8.53 & 4.07 & $\begin{array}{c}\text { Trabaja en } \\
\text { una empresa }\end{array}$ & 24.75 & 27.07 & 14.18 & 9.53 & 5.99 \\
\hline $\begin{array}{c}\text { Trabaja en el } \\
\text { gobierno }\end{array}$ & 22.68 & 22.84 & 14.80 & 8.35 & 4.20 & $\begin{array}{c}\text { Trabaja en el } \\
\text { gobierno }\end{array}$ & 25.46 & 27.67 & 14.38 & 9.53 & 6.38 \\
\hline $\begin{array}{l}\text { Trabaja por } \\
\text { su cuenta }\end{array}$ & 22.49 & 22.96 & 15.07 & 8.40 & 3.87 & $\begin{array}{l}\text { Trabaja por } \\
\text { su cuenta }\end{array}$ & 24.54 & 26.86 & 13.91 & 9.54 & 5.89 \\
\hline $\begin{array}{l}\text { Se dedica al } \\
\text { hogar }\end{array}$ & 20.35 & 23.08 & 14.76 & 8.99 & 3.89 & $\begin{array}{l}\text { Se dedica al } \\
\text { hogar }\end{array}$ & 29.31 & 33.49 & 17.08 & 12.02 & 7.55 \\
\hline
\end{tabular}




\begin{tabular}{|c|c|c|c|c|c|c|c|c|c|c|c|}
\hline \multicolumn{6}{|c|}{ Tienes hijos } & \multicolumn{6}{|c|}{ Tienes hijos } \\
\hline $\mathrm{Si}$ & 22.72 & 23.26 & 14.91 & 8.64 & 4.21 & $\mathrm{Si}$ & 21.51 & 21.77 & 12.93 & 7.83 & 4.96 \\
\hline No & 23.58 & 25.03 & 14.57 & 8.94 & 4.64 & No & 24.54 & 22.24 & 12.83 & 7.79 & 4.95 \\
\hline \multicolumn{6}{|c|}{ Vida sexual activa } & \multicolumn{6}{|c|}{ Vida sexual activa } \\
\hline $\mathrm{Si}$ & 26.54 & 26.11 & 13.59 & 9.21 & 6.37 & $\mathrm{Si}$ & 25.67 & 24.70 & 12.48 & 8.90 & 6.05 \\
\hline No & 26.94 & 26.35 & 13.55 & 9.03 & 6.98 & No & 25.99 & 25.96 & 13.23 & 9.33 & 6.47 \\
\hline \multicolumn{6}{|c|}{ Utilizas método anticonceptivo } & \multicolumn{6}{|c|}{ Utilizas método anticonceptivo } \\
\hline $\mathrm{Si}$ & 26.19 & 26.69 & 13.06 & 9.08 & 5.48 & $\mathrm{Si}$ & 28.16 & 28.21 & 13.73 & 8.90 & 6.70 \\
\hline No & 24.86 & 25.85 & 13.89 & 9.21 & 5.49 & No & 29.70 & 29.23 & 13.93 & 9.19 & 6.99 \\
\hline \multicolumn{6}{|c|}{ Tipo de método anticonceptivo } & \multicolumn{6}{|c|}{ Tipo de método anticonceptivo } \\
\hline Condón & 25.08 & 26.21 & 13.46 & 9.89 & 5.69 & Condón & 28.48 & 27.67 & 13.35 & 8.77 & 7.05 \\
\hline Pastillas & 25.16 & 25.37 & 13.95 & 9.13 & 5.15 & Pastillas & 28.28 & 26.89 & 12.14 & 8.28 & 7.10 \\
\hline Inyecciones & 27.76 & 26.94 & 13.08 & 8.95 & 5.71 & Inyecciones & 31.25 & 31.56 & 14.98 & 10.18 & 7.79 \\
\hline Ritmo & 25.91 & 24.16 & 12.66 & 8.65 & 5.09 & Ritmo & 28.73 & 27.50 & 13.14 & 8.48 & 7.38 \\
\hline Otros & 24.76 & 25.73 & 13.43 & 9.85 & 4.80 & Otros & 27.13 & 25.67 & 12.06 & 7.72 & 6.71 \\
\hline \multicolumn{6}{|c|}{ Cuenta con trabajo } & \multicolumn{6}{|c|}{ Cuenta con trabajo } \\
\hline $\mathrm{Si}$ & 23.96 & 24.93 & 13.67 & 9.29 & 6.59 & $\mathrm{Si}$ & 29.16 & 30.18 & 14.76 & 9.62 & 7.88 \\
\hline No & 24.85 & 25.83 & 13.57 & 9.53 & 6.90 & No & 29.25 & 30.14 & 14.96 & 9.51 & 8.17 \\
\hline \multicolumn{6}{|c|}{ Edad para convertirse en madre } & \multicolumn{6}{|c|}{ Edad para convertirse en madre } \\
\hline 12 a 15 años & 23.02 & 23.24 & 13.40 & 9.14 & 6.17 & 12 a 15 años & 28.81 & 27.63 & 13.18 & 8.41 & 7.58 \\
\hline 16 a 19 años & 24.55 & 24.20 & 14.39 & 8.95 & 5.94 & 16 a 19 años & 26.42 & 26.94 & 13.00 & 9.56 & 7.35 \\
\hline 20 a 23 años & 26.32 & 26.51 & 13.75 & 9.45 & 6.21 & 20 a 23 años & 29.38 & 27.71 & 13.49 & 9.06 & 6.82 \\
\hline 24 a 27 años & 26.26 & 26.93 & 14.31 & 9.49 & 6.22 & 24 a 27 años & 27.70 & 26.54 & 12.68 & 7.90 & 6.70 \\
\hline 28 a 31 años & 26.12 & 26.89 & 13.89 & 9.57 & 5.78 & 28 a 31 años & 28.19 & 27.09 & 12.94 & 8.03 & 6.52 \\
\hline 32 a 35 años & 26.29 & 26.84 & 13.72 & 9.53 & 5.53 & 32 a 35 años & 27.65 & 27.22 & 12.86 & 8.15 & 6.70 \\
\hline 36 a 39 años & 27.87 & 27.94 & 13.21 & 10.15 & 5.86 & 36 a 39 años & 30.00 & 30.16 & 13.59 & 9.23 & 7.20 \\
\hline Más de 40 & 29.10 & 29.88 & 15.21 & 10.93 & 5.83 & Más de 40 & 31.20 & 30.45 & 14.11 & 9.28 & 7.50 \\
\hline \multicolumn{6}{|c|}{ La escuela ofrece anticonceptivos } & \multicolumn{6}{|c|}{ La escuela ofrece anticonceptivos } \\
\hline $\mathrm{Si}$ & $26 . .57$ & 27.57 & 15.44 & 10.25 & 4.89 & $\mathrm{Si}$ & 26.88 & 27.41 & 12.54 & 8.53 & 6.16 \\
\hline No & 26.59 & 26.13 & 15.91 & 9.19 & 5.12 & No & 27.40 & 27.44 & 13.29 & 8.04 & 6.51 \\
\hline \multicolumn{6}{|c|}{ Dinero que gasta en anticonceptivos } & \multicolumn{6}{|c|}{ Dinero que gasta en anticonceptivos } \\
\hline No gasto & 26.20 & 26.15 & 13.97 & 9.28 & 6.19 & No gasto & 27.02 & 26.16 & 12.28 & 7.83 & 6.65 \\
\hline De 50 a 100 & 26.09 & 26.07 & 13.77 & 9.14 & 5.91 & De 50 a 100 & 27.59 & 26.34 & 12.88 & 7.79 & 6.42 \\
\hline De 100 a 150 & 26.40 & 26.92 & 14.07 & 9.84 & 6.06 & De 100 a 150 & 27.27 & 26.25 & 12.79 & 8.00 & 6.62 \\
\hline De 150 a 200 & 27.71 & 27.51 & 13.67 & 9.65 & 6.51 & De 150 a 200 & 27.67 & 28.08 & 13.61 & 8.44 & 6.54 \\
\hline De 200 a 250 & 25.66 & 26.81 & 13.19 & 9.87 & 5.52 & De 200 a 250 & 28.69 & 27.96 & 12.75 & 8.93 & 7.05 \\
\hline Más de 250 & 25.56 & 27.80 & 14.54 & 10.30 & 6.38 & Más de 250 & 29.11 & 27.94 & 13.29 & 8.92 & 7.56 \\
\hline
\end{tabular}

Fuente: Elaboración propia a partir de los resultados obtenidos del software SPSS.

Source: Own construction from the results obtained from the SPSS software. 


\section{Factor 2. La maternidad y el entorno universitario.}

La prueba de U de Mann Whitney, permitió detectar que existen diferencias en los grupos formados por mujeres y hombres; los hombres registraron mayores rangos promedios (1 216.29) en las creencias que miden la maternidad y el entorno universitario. De igual forma se aplicó la prueba no paramétrica de U de Mann Whitney con los grupos de estudiantes con hijos y sin hijos ( $\mathrm{P}>$ 0.05), hallándose que el grupo que no tienen hijos son los que calificaron más alto el factor (1184.29) (Tabla 2). De acuerdo con la prueba de contraste de Omnibus, se halló que tanto en el grupo de mujeres y de hombres se encontraron variables que abonan a la explicación del factor que analiza la maternidad y el entorno universitario $(\mathrm{P}<0.05)$. La prueba de los efectos del modelo permitió encontrar que en el caso de las mujeres la variable de tener hijos, contar con empleo y la escuela ofrece anticonceptivos, son las que abonaron más al factor $(\mathrm{P}<0.05)$. Y en el caso de los hombres, las variables con efectos fueron la ocupación de la madre, la ocupación del padre y tener hijos $(\mathrm{P}<0.05)$ (Tabla 3).

El análisis de las medias de las categorías de las variables anteriormente identificadas arrojó que las mujeres (25.03) y hombres (22.24) que no tenían hijos fueron quienes le otorgaron mayor puntuación al factor de las creencias de que se obstaculiza la vida profesional y académica de las mujeres que se embarazan o son madres. De igual forma las mujeres que no tienen empleo (25.83) y las que indicaron que la escuela si les ofrece métodos anticonceptivos (27.57) fueron quienes calificaron alto las creencias. También resultaron altas las puntuaciones en los hombres, quienes sus madres trabajaban por su cuenta (29.56), o que sus padres se dedicaban al hogar al momento del estudio (33.49) (Tabla 4).

La parte dos del modelo lineal permitió detectar que según la prueba de contraste de Omnibus arrojó que en los grupos de mujeres y de hombres existen variables que coadyuvan al entendimiento del factor $(\mathrm{P}<0.05)$. En el caso del grupo de mujeres, los efectos principales se dieron en si cuentan con trabajo y si la escuela proporciona anticonceptivos $(\mathrm{P}<0.05)$. En el caso de los hombres, se detectó en las variables de vida sexual activa, utiliza algún método anticonceptivo, tipo de anticonceptivo, cuentas con trabajo, edad para convertirse en madre y dinero que se gasta en anticonceptivos $(\mathrm{P}<0.05)$.

En el grupo de mujeres, se encontró que aquellas que manifestaron no tener una actividad laboral (25.83), fueron las que le dieron mayor cantidad de puntos a las creencias sobre la maternidad y el entorno universitario, caso contrario sucedió con las mujeres que argumentaron 
estar trabajando (24.93). De igual forma, se halló que las mujeres que dijeron que la escuela si proporciona los anticonceptivos fueron quienes están de acuerdo con estas creencias (27.57). Por lo que respecta al grupo de los varones, se halló que los que calificaron más alto el factor de la maternidad y el entorno universitario, fueron aquellos sin una vida sexual activa (25.96); lo mismo sucedió con los varones que manifestaron no usar anticonceptivos (29.23); y con el grupo de hombres que revelaron estar usando un método anticonceptivo y quienes dijeron que su pareja usaba inyecciones hormonales (31.56), así como con los jóvenes que manifestaron tener un empleo al momento del estudio (30.18). También, los que puntearon alto el factor fueron aquellos varones que consideraron que la edad ideal para ser madre es tener más de 40 años (31.20).

\section{Factor 3. La maternidad y los programas sociales.}

De acuerdo a la prueba estadística de U de Mann Whitney, se encontró que no existe diferencia entre los grupos formados por las mujeres y los hombres $(\mathrm{P}>0.05)$ (Tabla 2). Por otra parte, en los modelos lineales generalizados, en la primera parte, se encontró que solamente en el grupo de los hombres se obtuvo como variable con efectos la de ocupación del padre $(\mathrm{P}<0.05)$ (Tabla 3).

Los jóvenes, quienes dijeron que sus padres se dedicaban al hogar al momento del estudio (17.08), fueron quienes calificaron más alto el factor de las creencias sobre la maternidad y los programas sociales, lo que significa que simpatizan con la creencia de que el gobierno es el responsable de la atención médica de las mujeres embarazadas, de las políticas de conciliación en el trabajo, y el apoyo económico que necesitan las embarazadas; sin embargo, aquellos que sus padres trabajaban en la empresa (14.18), fueron los que no estuvieron del todo de acuerdo con los apoyos hacia las mujeres embarazadas.

En la parte dos del modelo y de acuerdo con la prueba de contraste de Omnibus, se halló nuevamente que solo en el grupo de los hombres hay variables con efectos hacia el modelo ( $\mathrm{P}<$ 0.05). La prueba de los efectos del modelo lineal generalizado en el grupo de los hombres encontró que las variables de vida sexual activa, si se ocupa métodos anticonceptivos, tipo de método anticonceptivo, la escuela ofrece anticonceptivos y la cantidad de dinero que gastas en anticonceptivos $(\mathrm{P}<0.05)$, son las que permiten interpretar el factor (Tabla 3).

Los datos que se obtuvieron de las medias por las categorías de las variables arrojaron que los hombres que calificaron alto sus creencias sobre la maternidad y los programas sociales fueron quienes señalaron no tener una vida sexual activa (13.23). De igual forma, ocurre con el grupo de 
los varones, que indicaron no estar haciendo uso de algún método anticonceptivo (13.93). Por otra parte, el grupo de hombres que señalaron estar usando un método anticonceptivo y que sus parejas empleaban las inyecciones (14.98), están de acuerdo con el trabajo que se pretende realizar desde los programas sociales. Así mismo, aquellos jóvenes que revelaron que la escuela no ofrece anticonceptivos dieron mayores puntos (13.29), y lo mismo sucedió con aquellos que manifestaron que gastaban en anticonceptivos, entre \$ 150 a $\$ 200$ pesos mexicanos al mes (13.61) (Tabla 4).

\section{Factor 4. La universidad y los programas de apoyo orientados a la maternidad.}

Este factor integra los elementos que analizan las estrategias de la universidad para prevenir y apoyar a las mujeres embarazadas o que son madres. Se aplicó la prueba de U de Mann Whitney y se encontró que existe diferencia $(\mathrm{P}<0.05)$ entre los grupos formados por las mujeres y los hombres; de acuerdo con los rangos promedios, los hombres calificaron más alto el factor que las mujeres. También se corrió la prueba con los grupos de jóvenes con y sin hijos, encontrándose diferencia entre ellos $(\mathrm{P}<0.05)$, los estudiantes que no tienen hijos son los que obtuvieron el promedio más alto (1183.40) con relación al factor (Tabla 2).

Se trabajó con los modelos lineales generalizados, la prueba de contraste de Omnibus arrojó que en el grupo de los hombres las variables con efectos hacia el factor fueron; la ocupación del papá y tener hijos ( $\mathrm{P}<0.05)$ (Tabla 3). Por su parte, el análisis de las medias por las categorías de las variables identificadas arrojó que los hombres que calificaron más alto las creencias del factor de la universidad y los programas sociales fueron los que dijeron tener hijos (7.83), al igual que los jóvenes cuyos padres se dedicaban al hogar al momento del estudio (12.02).

En la parte dos del modelo, se encontraron variables con efectos en los grupos de las mujeres y los hombres $(\mathrm{P}<0.05)$. En el grupo de mujeres, se detectaron las variables de; si cuentan con trabajo, la escuela te proporciona anticonceptivos y el dinero que invierten al mes para adquirir anticonceptivos $(\mathrm{P}<0.05)$. En el grupo de los hombres, se encontraron las variables de en el uso de anticonceptivos, edad para convertirse en madre, la escuela ofrece anticonceptivos y el dinero que gastan en anticonceptivos al mes (P < 0.05) (Tabla 3).

$\mathrm{El}$ análisis de las medias por las categorías de las variables que permiten explicar el factor se halló que, en el grupo de mujeres, se encontró que aquellas que dijeron estar desempleadas (9.53), fueron las que calificaron de mejor forma las acciones de la escuela; lo mismo ocurrió con las mujeres que manifestaron que la escuela les ofrece anticonceptivos (10.25). Por último, las 
mujeres que señalaron que gastaban más de $\$ 250$ pesos mexicanos al mes en anticonceptivos fueron las que otorgan más puntos positivos al trabajo de la universidad (10.30) (Tabla 4).

En el grupo de los hombres, se encontró que quienes ocupaban el anticonceptivo de las inyecciones calificaron el factor alto (10.18); aparte, los jóvenes que señalaron que la escuela los dotaba de anticonceptivos (8.53) fueron los que dijeron que, de igual forma, estaban de acuerdo con el trabajo de la universidad, lo mismo ocurrió con los hombres que revelaron que gastaban al mes en anticonceptivos un promedio de \$ 200 a $\$ 250$ pesos mexicanos (8.93).

\section{Factor 5. El aborto.}

La prueba estadística de U de Mann Whitney permitió encontrar diferencias entre los grupos de mujeres y de hombres $(\mathrm{P}<0.05)$, siendo los hombres quienes estuvieron de acuerdo con la creencia de que las mujeres que abortan deben ser castigadas por la ley, y que las mujeres jóvenes no tienen derecho abortar porque fue su responsabilidad quedar embarazadas. De igual forma se corrió la prueba U de Mann Whitney con los grupos formados de estudiantes que tienen hijos y los que no, encontrándose que no existe diferencia entre ellos ( $\mathrm{P}>0.05)$ (Tabla 2). Se trabajó con los modelos lineales generalizados, y de acuerdo con la prueba de Omnibus se encontró que en el grupo de hombres es donde se tiene variables con efectos $(\mathrm{P}<0.05)$ (Tabla 3). La prueba de los efectos del modelo presentó variación en la variable que mide la ocupación del papá. Los jóvenes que indicaron que su padre se dedicaba al hogar calificaron alto el factor que mide el aborto (7.55) (Tabla 4).

\section{Discusión}

En este trabajo se encontró que, con respecto a la percepción de la maternidad y el mercado laboral, la población de los hombres estudiantes fueron los que percibieron más alto las creencias que desfavorecen el empleo a las mujeres jóvenes que están embarazadas o que son madres. Por su parte, cuando se trabajaron los modelos lineales generalizados, los hombres que señalaron que sus madres o sus padres se dedican al hogar, otorgaron mayor valor al factor. Lo anterior tiene implicaciones complejas para las estudiantes que son madres porque esas creencias las pueden limitar ya sean en su acceso o ascenso en el mercado laboral, al parecer los varones le siguen otorgando a las mujeres la responsabilidad del cuidado de los hijos por encima de su desarrollo profesional. Por su parte, Berthelon y Kruger (2017), encontraron que las mujeres que llegan a ser 
madres en su juventud son afectadas en sus avances de carrera académica, y debido a que son menos los años de educación que logran cursar, esto se refleja en las oportunidades laborales que pueden alcanzar, lo que induce a una situación de pobreza, por lo que es necesario reforzar las políticas públicas orientadas a reducir la maternidad temprana en jóvenes. De igual forma, Güngör y Biernat (2009), documentaron en su estudio con jóvenes universitarios que solicitan empleo, estos reflejan un sesgo de género para las mujeres que son madres, ya que se les considera como menos comprometidas, con escasa seguridad en sí mismas y con menos oportunidades de ser contratadas; de igual forma, en su investigación enfatizan que las oportunidades de empleo para las madres solteras son más complicadas penalizándolas severamente por maternidad. Al respecto, Joona (2018), señaló que es necesario cambiar la imagen de las mujeres que son madres en el sector laboral, por lo que se requiere trabajar con todas las iniciativas posibles para incorporarlas al sector educativo.

Por otro lado, se tiene evidencia con estudios realizados por Chung (2018), que las mujeres que son madres piensan que el hecho de contar con trabajos de tiempo parcial les resta posibilidades de ascensos y promociones en su trabajo, además identifican que la sociedad engendra una percepción negativas hacia el compromiso de las madres con su desempeño y productividad, en definitiva, son las mujeres las que perciben mayor discriminación en el sector laboral. Lo anterior se contrapone a lo expuesto por Chung (2018), ya que en esta investigación se encontró que las mujeres que reportaron una actividad laboral, ellas percibieron menos las creencias que desfavorecen la maternidad y el empleo femenino, esto puede ser derivado de que ellas tengan contacto con otras mujeres que ya ejerzan la maternidad y que son capaces de combinarla con el trabajo y la vida académica de forma equilibrada. Siguiendo con los resultados del factor de la maternidad, en la investigación se encontró que 81 mujeres y 74 hombres dijeron tener hijos, tantas mujeres (48\%) y hombres (55\%) dijeron estar de acuerdo con que el mercado laboral favorece a las mujeres que no tienen hijos sobre las que si los tienen. Por otro lado, el 35\% de mujeres y el $44 \%$ de hombres estuvieron de acuerdo con que por su nivel de competencia no contempla oportunidades de empleo a las madres jóvenes. Los hallazgos permiten entender que los jóvenes que son padres en diversos casos mantienen la creencia de que la maternidad es un obstáculo para la inserción y permanencia en el mercado de trabajo.

Por otra parte, Milewski et al. (2018), recomiendan trabajar con iniciativas que permitan impulsar el empleo femenino, además de fortalecer las políticas que permitan conciliar la vida 
laboral y familiar, de esta forma, es posible mantener por más tiempo ocupadas laboralmente a las mujeres. De acuerdo con la Comisión Económica para América Latina y el Caribe se requieren reformas estructurales para que ellas se mantengan en el mercado laboral y en la escuela, es imprescindible que cuenten con el apoyo de redes de guarderías y escuelas de tiempo completo de calidad subsidiadas por el gobierno (Giddings et al., 2004; Casas y Herrera, 2012; Pacelli et al., 2013; Cuesta y Cancian, 2015; Hazan y Zoabi, 2015; Apps et al., 2016). Los gobiernos necesitan establecer estrategias con los empleadores para que no pierdan la confianza en las mujeres que son madres y resten habilidades y capacidades, de esta forma se puede garantizar que las mujeres accedan a los recursos económicos y así abatir la pobreza y la precariedad en los empleos (Edwards, 2005; Ciciolla et al., 2017; Milewski et al., 2018).

Con relación a la maternidad y el entorno universitario, se halló que fueron los hombres los que percibieron con un valor alto el factor, Por lo que respecta a las estudiantes que tienen hijos, el $43 \%$ de ellas y el $49 \%$ de ellos consideran que es evidente el abandono de los estudios universitarios de parte de las madres solteras, aunado a que el 32\% de las mujeres y $45 \%$ de hombres piensa que las mujeres que son madres o que están embarazadas es difícil que logren destacar en clase a pesar de contar con la inteligencia y habilidades ya que es evidente su falta de tiempo, además el 33\% de ellas y el 39\% de ellos reconoce que los profesores discriminan a las mujeres por estar embarazadas. Las instituciones que participaron en el estudio no cuentan con programas orientados abatir el abandono escolar de parte de las universitarias que son madres. Por lo anterior, es evidente que las estudiantes reconocen que es posible que no se logre combinar los estudios universitarios con la maternidad y más en aquellas mujeres que son madres solteras. Entonces la maternidad para quienes los jóvenes que son padres es un obstáculo para continuar con su formación académica.

Se tiene evidencia de que las guarderías para estudiantes que han instalado universidades como la Autónoma de Tamaulipas, la Autónoma de Querétaro y la Autónoma de Chiapas, por mencionar algunas, han permitido disminuir el abandono escolar de los estudiantes que tienen hijos. En el caso de la Autónoma de Tamaulipas no tiene costos para los jóvenes, atienden a un máximo de 150 menores y están desde el 2012 operando (González, 2019). La estancia que opera en la Universidad Autónoma de Querétaro, tienen un costo de \$600 pesos al mes para los jóvenes, pueden cuidar hasta 60 niños, pero esperan ampliar las instalaciones para poder atender a unos 120 niños (Colón, 2019). Por último se obtuvo el dato de la Universidad Autónoma de Chiapas, tienen 
dos guarderías, la primera está en Tapachula y la segunda en Tuxtla, atienden aproximadamente 70 niños y el costo es de $\$ 200$ pesos al mes (Rubio, 2019).

De acuerdo con Behboodi et al. (2017); Chambers y Erausquin (2018), en las universidades se necesita reflexionar y sensibilizar sobre los roles de las madres que son estudiantes para eliminar algunas expresiones que discriminan su condición y que las lleva al posible desgaste emocional. Además, los tomadores de decisiones deben desarrollar estrategias encaminadas en brindar apoyo para que no abandonen la escuela y continúen con sus procesos de adquisición de conocimientos con programas escolares flexibles, porque la educación significa desarrollo y progreso para ellas. $\mathrm{Al}$ respecto, autores como Vinson y Stevens (2014), argumentaron que la escuela tiene que tomar medidas para que los estudiantes, personal docente y autoridades no avergüencen, hostiguen, estigmaticen o discriminen a las estudiantes embarazadas o que son madres.

Con relación al factor que evalúa la percepción que tienen los estudiantes sobre la universidad y los programas de prevención y apoyo a la maternidad, se encontró que las mujeres calificaron más alto los elementos positivos que le otorgan a la universidad con respecto al tema de la prevención del embarazo. Sin embargo, la universidad únicamente cuenta con una dotación de preservativos que otorga la Secretaría de Salud. Al respecto, se puede trabajar de forma intensa con los jóvenes y dotarlos de información sobre anticonceptivos, pero, de acuerdo con Jones et al. (2019) es necesario sensibilizar a los profesionales que están cerca de las madres y padres jóvenes, ellos deben estar conscientes de la discriminación, exclusión y aislamiento que sufren los jóvenes, aunado a que los responsables de la elaboración de políticas garanticen que las mujeres recibirán apoyo formal y profesional que les permitan acceder a oportunidades de apoyo.

El factor que evalúa la percepción con relación a la maternidad y los programas sociales se halló que los hombres que no tienen una vida sexual activa son quienes otorgan más puntos a las creencias. Los hombres que indicaron no contar con una vida sexual activa, se encontró que el 58\% cree que el gobierno debe hacerse cargo de la asistencia médica de las madres y de sus hijos, aunado a que el $64 \%$ de ellos considera que es necesario que las empresas mantengan políticas de conciliación familiar y laboral y el $45 \%$ conoce los programas sociales dirigidos a las madres solteras. Sin embargo, se halló que los varones cuyos padres trabajan en la empresa son los que no estuvieron de acuerdo con los programas sociales orientados a la maternidad, caso contrario sucede con aquellos alumnos que sus padres se dedicaban al hogar en el momento del estudio. Lo anterior, 
tiene implicaciones importantes, porque se requieren no solo programas dirigidos a las madres sino también a los padres.

El factor que analizó las creencias sobre la universidad y los programas de apoyo orientados a la maternidad, encontró que los hombres que tienen hijos son los que dieron más puntos, se identificó que el $44 \%$ de ellos está de acuerdo con que las mujeres que están embarazadas en todo momento están protegidas por la escuela y que ésta las orienta. Al parecer, los apoyos de parte de la escuela se hacen visibles en el momento en que los estudiantes lo requieren, sin embargo, se requiere profundizar para conocer si solo se ofrece asesoría con respecto al seguro médico, porque en el momento de la investigación no se tiene ubicado algún programa específicamente desarrollado para apoyar a las mujeres que están embarazadas o que son mamás. De acuerdo con Jones et al. (2019) es necesario sensibilizar a los profesionales que están cerca de las madres y padres jóvenes, ellos necesitan ser conscientes de la discriminación, exclusión y aislamiento que sufren los jóvenes, aunado a que los responsables de la elaboración de políticas garanticen que las mujeres recibirán apoyo formal y profesional que les permitan acceder a oportunidades de apoyo.

Por último, el factor que estudia las percepciones de los jóvenes sobre el aborto, los resultados señalan que los hombres son los que calificaron alto las creencias del aborto. Al respecto, Sharp, Richter y Rutherford (2015) mencionan que a pesar de que los hombre carecen de prerrogativas legales en la decisión de interrumpir el embarazo, la ley si establece sus responsabilidades de manutención, por lo anterior, enfatizan que la educación sexual previene que la paternidad involuntaria aparezca en los hombres, Además, en la investigación se halló que el $26 \%$ de los estudiantes que tienen hijos estuvieron de acuerdo con la creencia de que las mujeres no tienen derecho de abortar porque fue su responsabilidad el quedar embarazadas y por lo tanto deben asumirla, de igual forma el $26 \%$ de los jóvenes que son padres indicaron estar de acuerdo con que las mujeres que abortan deben ser castigadas por la ley por considerarse un delito. Sin embargo, el tema es complejo y polémico, por lo tanto, se necesita investigar a mayor detalle el fenómeno.

\section{Conclusiones}

Se concluye que los hombres que participaron en el estudio siguen manteniendo creencias relacionadas con que la maternidad es un obstáculo para el acceso y desarrollo profesional de las mujeres. En la cuestión académica, también tuvieron una mayor percepción de que las mujeres que 
son madres en la etapa de estudiantes, pueden abandonar sus estudios y cortar su futuro profesional. Lo anterior, puede deberse a que los varones mantengan la creencia, de que la responsabilidad de la crianza es de las mujeres, lo cual, acarrea diversas implicaciones que pueden reflejarse en la dedicación de las actividades en el hogar de parte de las mujeres, dejando de lado su formación académica. Se concluye que las instituciones requieren trabajar en la sensibilización de todos los actores para evitar el abandono escolar, por la falta de flexibilidad o apoyo económico a las mujeres embarazadas o futuros padres, jefes o jefas de familia. Primero, se necesita la elaboración de un censo, para conocer cuántos hombres y cuántas mujeres están en dichas situaciones. Por otro lado, es viable que las instituciones continúen con los programas de prevención del embarazo, ya que tanto las mujeres como los hombres que señalaron que la escuela les ofrece anticonceptivos, calificaron favorablemente ese tipo de acciones. Además, se recomienda trabajar con talleres enfocados a la educación y salud sexual, de esa forma, los jóvenes tendrán la posibilidad de elegir en el momento en que ellos decidan convertirse en madres y padres.

El panorama para las mujeres en el mercado de trabajo es complicado y más cuando son madres o se encuentran embarazadas, sumando a que muchas de las trabajadoras pueden estar estudiando. Se concluye que es necesario trabajar en políticas que coadyuven a la conciliación entre la vida laboral y familiar, además de considerar la estructura de un sistema de cuidados y asistencia que permita a las mujeres gozar de las mismas oportunidades laborales de los hombres. Además de mantener una vigilancia para las empresas sobre las obligaciones que tienen con las mujeres durante su proceso de embarazo y después del mismo.

\section{$\underline{\text { Agradecimientos }}$}

Al Instituto Politécnico Nacional a través de la Secretaria de Investigación y Posgrado. Al Instituto

Tecnológico de Gustavo A. Madero por las facilidades otorgadas para la aplicación de los instrumentos.

\section{$\underline{\text { Referencias }}$}

Allen, J., Gamble, J., Stapleton, H., \& Kildea, S. (2012). Does the way maternity care is provided affect maternal and neonatal outcomes for young women? A review of the research literature. Women and Birth. 25(2): 54-63. 
Amuedo-Dorantes, C. \& Kimmel, J. (2005). The motherhood wage gap for women in the United States: The importance of college and fertility delay. Review of Economics of the Household. 3(1): 17-48.

Aponte, M. R. E. \& Correa, D. R. V. (2012). Calidad de vida de madres adolescentes estudiantes universitarias. Revista Colombiana de Psiquiatría. 41(3): 536-549.

Apps, P., Kabátek, J., Rees, R., \& van Soest, A. (2016). Labor supply heterogeneity and demand for child care of mothers with young children. Empirical Economics. 51(4): 1641-1677.

Arceo-Gómez, E. O. \& Campos-Vazquez, R. M. (2014). Teenage pregnancy in Mexico: Evolution and consequences. Latin American Journal of Economics. 51(1): 109-146.

Atienzo, E. E., Campero, L., Lozada, A. L. \& Herrera, C. (2014). Aspiraciones educativas y familiares como condicionantes en la prevención de embarazos tempranos en México. Salud Pública de México. 56(3): 286-294.

Baranowska-Rataj, A., Matysiak, A., \& Mynarska, M. (2014). Does lone motherhood decrease women's happiness? Evidence from qualitative and quantitative research. Journal of Happiness Studies. 15(6): 1457-1477. DOI: https://doi.org/10.1007/s10902-013-9486-Z

Behboodi, Z., Ordibeheshti-Khiaban, M., Esmaeili, M., \& Salsali, M. (2017). Motherhood challenges and well-being along with the studentship role among Iranian women: A qualitative study. International Journal of Qualitative Studies on Health and WellBeing. 12(1): 1335168.

Berthelon, M. \& Kruger, D. I. (2017). Does adolescent motherhood affect education and labor market outcomes of mothers? A study on young adult women in Chile during 19902013. International Journal of Public Health. 62(2): 293-303.

Breen, A. V. \& McLean, K. C. (2010). Constructing resilience: Adolescent motherhood and the process of self-transformation. In K. McLean and M. Pasupathi (Eds.), Narrative development in adolescence (pp. 151-168). Boston, MA: Springer US.

Casas, L. \& Herrera, T. (2012). Protección de la maternidad versus derechos de maternidad para las trabajadoras en Chile: una reseña histórica. Reproductive Health Matters. 20(40): 139147. DOI: 10.1016/S0968-8080(12)40666-8

Colón, F. (2019) Responsable de la estancia infantil para estudiantes de la Universidad Autónoma de Querétaro, México. 
Naciones Unidas (1979) Convención sobre la eliminación de todas las formas de discriminación contra la mujer. [En línea]. Disponible en: https://www.ohchr.org/SP/ProfessionalInterest/Pages/CEDAW.aspx. Fecha de consulta: 30 de septiembre de 2019.

Cuesta, L. \& Cancian, M. (2015). The effect of child support on the labor supply of custodial mothers participating in TANF. Children and Youth Services Review. 54: 49-56.

Chambers, B. D. \& Erausquin, J. T. (2018). Reframing the Way We Think About Teenage Motherhood. In S. Choudhury, J. Toller, and M. Withers (Eds.), Global Perspectives on Women's Sexual and Reproductive Health Across the Lifecourse (pp. 59-71). Switzerland: Springer, Cham.

Chung, H. (2018). Gender, Flexibility Stigma and the Perceived Negative Consequences of Flexible Working in the UK. Social Indicators Research. 1-25.

Ciciolla, L., Curlee, A. S., \& Luthar, S. S. (2017). What Women Want: Employment Preference and Adjustment Among Mothers. Journal of Family and Economic Issues. 38(4): 494-513.

De-Hoon, S., Keizer, R., \& Dykstra, P. (2017). The influence of motherhood on income: do partner characteristics and parity matter? Community, Work \& Family. 20(2): 211-225.

Devos, T., Viera, E., Diaz, P., \& Dunn, R. (2007). Influence of motherhood on the implicit academic self-concept of female college students: Distinct effects of subtle exposure to cues and directed thinking. European Journal of Psychology of Education. 22(3): 371.

De Beauvoir, S. (1981). El segundo sexo (1949). Buenos Aires: Siglo XX.

Donath, O. (2016). Madres arrepentidas: una mirada radical a la maternidad y sus falacias sociales. Reservoir Books.

Drange, N. \& Rege, M. (2013). Trapped at home: The effect of mothers' temporary labor market exits on their subsequent work career. Labour Economics. 24: 125-136.

Edwards, M. E. (2005). Occupational structure and the employment of American mothers of young children. Journal of Family and Economic Issues. 26(1): 31-53.

Ezawa, A. (2007). How Japanese single mothers work. Japanstudien. 18(1): 59-83.

Felfe, C. (2012). The motherhood wage gap: What about job amenities? Labour Economics. 19(1): 59-67.

DOI: https://econpapers.repec.org/scripts/redir.pf?u=https\%3A\%2F\%2Fdoi.org\%2F10.1016\%2 52Fj.labeco.2011.06.016;h=repec:eee:labeco:v:19:y:2012:i:1:p:59-67

$\mathrm{N}^{\circ}$ 26, Vol. 13 (2), 2021. ISSN 2007 - 0705, pp.: 1 - 29 - 25 - 
Frías, S. M. (2011). Hostigamiento, acoso sexual y discriminación laboral por embarazo en México. Revista mexicana de sociología, 73(2), 329-365.

Gamboa, L. F. \& Zuluaga, B. (2013). Is there a motherhood penalty? Decomposing the family wage gap in Colombia. Journal of family and economic issues. 34(4): 421-434.

García, I. A. (2015). Feminismos y maternidades en el siglo XXI. Dilemata, (18), 63-81.

Gash, V. (2009). Sacrificing their careers for their families? An analysis of the penalty to motherhood in Europe. Social Indicators Research. 93(3): 569-586.

Giddings, L., Dingeldey, I., \& Ulbricht, S. (2004). The commodification of lone mothers' labor: A comparison of US and German policies. Feminist Economics. 10(2): 115-142.

González, M. (2019) Responsible de la estancia infantil para estudiantes de la Universidad Autónoma de Tamaulipas, México.

Güngör, G. \& Biernat, M. (2009). Gender bias or motherhood disadvantage? Judgments of blue collar mothers and fathers in the workplace. Sex Roles. 60(3-4): 232-246.

Grunow, D. \& Aisenbrey, S. (2016). Economic instability and mothers' employment: A comparison of Germany and the US. Advances in Life Course Research. 29: 5-15.

Hazan, M. \& Zoabi, H. (2015). Do highly educated women choose smaller families? The Economic Journal. 125(587): 1191-1226.

Hoffnung, M. (2004). Wanting it all: Career, marriage, and motherhood during college-educated women's 20s. Sex roles. 50(9-10): 711-723.

Ibáñez Gracia, T. (2011). Introducción a la psicología social. Editorial UOC. España.

Jones, C., Whitfield, C., Seymour, J., \& Hayter, M. (2019). 'Other Girls': A Qualitative Exploration of Teenage Mothers' Views on Teen Pregnancy in Contemporaries. Sexuality \& Culture. $1-14$.

Joona, P. A. (2018). How does motherhood affect self-employment performance? Small Business Economics. 50(1): 29-54.

Lappegård, T. \& Rønsen, M. (2005). The multifaceted impact of education on entry into motherhood. European Journal of Population/Revue européenne de Démographie. 21(1), 31-49. DOI : https://doi.org/10.1007/s10680-004-6756-9

Lavee, E. \& Benjamin, O. (2016). "I've Got No Choice” Low-Income Mothers' Emotional Management of Caring Crisis. Journal of Family Issues. 37(7): 997-1021. 
Li, Y. (2017). The effects of formal and informal child care on the Mother's labor supply Evidence from urban China. China Economic Review. 44: 227-240.

Looze, J. (2014). Young women's job mobility: the influence of motherhood status and education. Journal of Marriage and Family. 76(4): 693-709.

Milewski, N., Struffolino, E., \& Bernardi, L. (2018). Migrant Status and Lone Motherhood-Risk Factors of Female Labour Force Participation in Switzerland. In L. Bernardi and D. Mortelmans (Eds.), Lone Parenthood in the Life Course (pp. 141-163). Switzerland: Springer, Cham.

Miller, A. R. (2011). The effects of motherhood timing on career path. Journal of Population Economics. 24(3): 1071-1100.

Morgenroth, T. \& Heilman, M. E. (2017). Should I stay or should I go? Implications of maternity leave choice for perceptions of working mothers. Journal of Experimental Social Psychology. 72: 53-56.

Myrskylä, M., Barclay, K., \& Goisis, A. (2017). Advantages of later motherhood. Der Gynäkologe. 50(10): 767-772.

Naciones Unidas Derechos Humanos, Oficina del Alto Comisionado (1966). Pacto Internacional de Derechos Civiles y Políticos. [En línea]. Disponible en: https://www.ohchr.org/sp/professionalinterest/pages/ccpr.aspx. Fecha de consulta: 11 de septiembre de 2020.

Narita, R. \& Diaz, M. D. M. (2016). Teenage motherhood, education, and labor market outcomes of the mother: Evidence from Brazilian data. Economia. 17(2): 238-252.

Noonan, M. C., Smith, S. S., \& Corcoran, M. E. (2007). Examining the impact of welfare reform, labor market conditions, and the Earned Income Tax Credit on the employment of black and white single mothers. Social Science Research. 36(1): 95-130.

Organización de los Estados Americanos (1979). Convención Americana sobre los Derechos Humanos, suscrita en la conferencia especializada Interamericana sobre Derechos Humanos. [En línea]. Disponible en: https://www.oas.org/dil/esp/tratados_b32_convencion_americana_sobre_derechos_humanos.htm. Fecha de consulta: 11 de septiembre de 2020.

Organización de las Naciones Unidas, ONU Mujeres (1979). Convención sobre la eliminación de todas las formas de discriminación contra la mujer (CEDAW). [En línea]. Disponible en: 
https://www.un.org/womenwatch/daw/cedaw/text/sconvention.htm. Fecha de consulta: 11 de septiembre de 2020.

Organización Internacional del Trabajo (1958). Convenio sobre discriminación (empleo y ocupación), número 111. [En línea]. Disponible en: https://www.ilo.org/dyn/normlex/es/f?p=NORMLEXPUB:12100:0::NO::P12100_ILO_C ODE:C111. Fecha de consulta: 11 de septiembre de 2020.

Pacelli, L., Pasqua, S., \& Villosio, C. (2013). Labor market penalties for mothers in Italy. Journal of Labor Research. 34(4): 408-432.

Petit, C., \& Graglia, S. (2005). Introducción a la psicología social. Manual para los estudios del turismo. Editorial Brujas. Argentina.

Rodríguez-Vignoli, J. (2017). Fecundidad no deseada entre las adolescentes latinoamericanas: un aumento que desafía la salud sexual y reproductiva y el ejercicio de derechos, Naciones Unidas. [En línea]. Disponible https://repositorio.cepal.org/bitstream/handle/11362/42511/1/S1700871_es.pdf. Fecha de consulta: 9 de mayo de 2018.

Rubio, F. (2019) Responsable de la estancias infantiles para estudiantes de la Universidad Autónoma de Chiapas, México.

Sánchez, A. G. C. (2015). La práctica social de la maternidad y de la paternidad: en jóvenes estudiantes de nivel superior: un acercamiento a las problemáticas cotidianas. Estudios sobre las Culturas Contemporáneas. (2): 103-123.

Stadelmann-Steffen, I. (2011). Dimensions of Family Policy and Female Labor Market Participation: Analyzing Group-Specific Policy Effects. Governance. 24(2): 331-357.

Stanfors, M. and Scott, K. (2013). Intergenerational transmission of young motherhood. Evidence from Sweden, 1986-2009. The History of the Family. 18(2): 187-208. DOI: https://doi.org/10.1080/1081602X.2013.817348

Staff, J. \& Mortimer, J. T. (2012). Explaining the motherhood wage penalty during the early occupational career. Demography. 49(1): 1-21.

Stafford, M., Lacey, R., Murray, E., Carr, E., Fleischmann, M., Stansfeld, S., \& McMunn, A. (2018). Work-family life course patterns and work participation in later life. European Journal of Ageing. 16(1): 83-94. 
Thomson, R. (2014). Expectations and Realities: Motherhood and the Female 'Choice'Biography. In J. Holland and R. Edwards (Eds.), Understanding Families Over Time (pp. 106-123). London: Palgrave Macmillan.

Viitanen, T. (2014). The motherhood wage gap in the UK over the life cycle. Review of Economics of the Household. 12(2): 259-276. DOI: https://doi.org/10.1007/s11150-012-9145-X

Villalobos-Hernández, A., Campero, L., Suárez-López, L., Atienzo, E. E., Estrada, F., \& la VaraSalazar, D. (2015). Embarazo adolescente y rezago educativo: análisis de una encuesta nacional en México. Salud Pública de México. 57(2): 135-143.

Vinson, J. \& Stevens, S. (2014). Preventing pregnancy or supporting students? Learning from the stories of young mothers. Sexuality Research and Social Policy. 11(4): 322-336.

Watts, M. C. N. C., Liamputtong, P., \& Mcmichael, C. (2015). Early motherhood: a qualitative study exploring the experiences of African Australian teenage mothers in greater Melbourne, Australia. BMC public health. 15(1): $873 . \quad$ DOI: https://doi.org/10.1186/s12889-015-2215-2

Watson, L. L. \& Vogel, L. R. (2017). Educational resiliency in teen mothers. Cogent Education. 4(1): 1-22.

Weed, K. \& Nicholson, J. S. (2015). Differential social evaluation of pregnant teens, teen mothers and teen fathers by university students. International journal of adolescence and youth. 20(1): 1-16.

Yerkes, M. (2010). Diversity in work: the heterogeneity of women's employment patterns. Gender, Work \& Organization. 17(6): 696-720. 